\title{
Network Oscillations: Emerging Computational Principles
}

\author{
Terrence J. Sejnowski ${ }^{1,2}$ and Ole Paulsen ${ }^{3,4}$ \\ ${ }^{1}$ Howard Hughes Medical Institute, Salk Institute for Biological Studies, La Jolla, California 92037, ${ }^{2}$ Department of Biology, University of California, San \\ Diego, La Jolla, California 92093, ${ }^{3}$ Department of Physiology, Anatomy, and Genetics, University of Oxford, Oxford OX1 3PT, United Kingdom, and ${ }^{4}$ Centre \\ for the Biology of Memory, Norwegian University of Science and Technology, NO-7489 Trondheim, Norway
}

Despite extensive work on the behavioral and physiological correlates of brain rhythms, it is still unresolved whether they have any important function in the mammalian cerebral cortex. In particular, there is no consensus on whether there are general computational roles for network oscillations. Three main possibilities will be discussed here. One possibility is that network oscillations contribute to representation of information. A second idea is that, rather than representing information as such, oscillations and synchrony regulate the flow of information in neural circuits. A third possibility is that oscillations assist in the storage and retrieval of information in neural circuits. These three possibilities are not mutually exclusive.

\section{Representation of sensory information}

Representation of information may be assisted by the tendency of oscillations to synchronize action potentials in principal cells (Singer and Gray, 1995). This potential function has been studied in detail in the visual system, in which synchronization by gamma oscillation has been suggested as a way to solve the "binding problem," i.e., how various sensory features can be bound into a coherent cognitive percept (Gray and Singer, 1989; Singer and Gray, 1995). Alternatively, or additionally, information could also be represented by oscillation phase. Thus, network oscillations may provide a temporal reference structure, relative to which spike times become meaningful.

A good example of phase encoding is "phase precession" in hippocampal place cells (O'Keefe and Recce, 1993). When a rodent traverses through a place field on a linear track, the place cell fires progressively earlier relative to the theta oscillation (O'Keefe and Recce, 1993). It has been suggested that, under these conditions, the phase of firing carries more information about the location of the animal than does the rate of firing (Tsodyks et al., 1996; Jensen and Lisman, 2000). However, the phase precession phenomenon is most prominent in linear environments and might not necessarily be more informative than firing rate in twoand three-dimensional space (Zhang et al., 1998). To what extent firing rate and spike phase covary or are separable entities during in vivo activity is currently debated (Harris et al., 2002; Mehta et al., 2002; Huxter et al., 2003).

\footnotetext{
Received Sept. 3, 2005; revised Dec. 2, 2005; accepted Dec. 17, 2005.

This work was supported by the Howard Hughes Medical Institute (T.J.S.), the Wellcome Trust (0.P.), and the Biotechnology and Biological Sciences Research Council, United Kingdom (0.P.).

Correspondence should be addressed to Dr. Terrence J. Sejnowski, Computational Neurobiology Laboratory, Howard Hughes Medical Institute, The Salk Institute for Biological Studies, 10010 North Torrey Pines Road, La Jolla, CA 92037.E-mail: terry@salk.edu.

D0I:10.1523/JNEUROSCI.3737-05d.2006

Copyright $\odot 2006$ Society for Neuroscience $\quad 0270-6474 / 06 / 261673-04 \$ 15.00 / 0$
}

\section{Spike-timing reliability}

A prerequisite for synchronization and phase codes to work is that the temporal precision of spike generation is sufficiently high and that action potential timing is reliable. Early work suggested that spike timing is highly variable from trial to trial (Rieke et al., 1997; Shadlen and Newsome, 1998), and it was until recently thought that the required precision is beyond the biophysical capability of cortical neurons (Softky, 1995). However, when more naturalistic fluctuating inputs are used, mammalian cortical neurons in vitro support highly precise and reliable spike times (Mainen and Sejnowski, 1995; Nowak et al., 1997). In vivo experiments have shown that cortical responses can be more reliable than previously thought (Buracas et al., 1998; Kara et al., 2000).

Reliability is greatest when the input driving frequency matches the intrinsic frequency preference of neurons (Pike et al., 2000; Fellous et al., 2001). Furthermore, downstream neurons must be able to detect synchronous activity for the activity to propagate in the network. Cortical neurons are good detectors of correlated activity (Salinas and Sejnowski, 2000), and dendrites have several interesting nonlinear properties that could exploit spike-time arrival (Mel, 1999). Encoding by phase and synchronization has highly attractive computational properties (Hopfield, 1995; Bazhenov et al., 2001a,b; Brody and Hopfield, 2003).

If oscillations are essential for binding together perceptual features, then it should be possible to disrupt perception by selectively interfering with the oscillations. This is a difficult experiment that may be possible using gene knock-out experiments in mice or viral vectors to target specific neurons in monkey cortex.

\section{Regulating the flow of information}

However, oscillations could have processing roles separate from the representation of information. Fast oscillations are enhanced during states of heightened alertness and attention. Thus, they may be involved in neuronal communication more than representation as such, i.e., they may regulate the strength rather than the content of neural signals (Salinas and Sejnowski, 2001). Several properties of neuronal networks would suggest such a role. First, network oscillations as seen in the EEG are state dependent and regulated by the behavioral task. Second, the emergence of oscillations depends on the neuromodulatory state of the network. Neuromodulators that regulate attention, such as acetylcholine, can themselves induce network oscillations (Fisahn et al., 1998). Third, the correlated activity of neurons increases the gain of neuronal integration.

Neurons are particularly sensitive to input correlation when the excitatory and inhibitory inputs are balanced, as seen during oscillatory states (Salinas and Sejnowski, 2000). Thus, the output 
of the neuron will be determined not only by the firing rates of its inputs but also by their correlations (Salinas and Sejnowski, 2001). The regulation of interaction strength with target neurons by coherence has been reported in corticospinal projections (Schoffelen et al., 2005). Thus, the oscillatory activity might serve as a dynamic nonlinear filter capable of selecting the salient and significant inputs to the network.

Perhaps the strongest evidence that oscillations and synchrony in the gamma band are involved in attention arises from experiments on awake, behaving monkeys, in which the visual input is kept fixed but the covert attention of the monkey is shifted to different parts of the visual scene. Increases were observed in the coherence between the spikes of single neurons in the visual cortex and local field potentials in the gamma band (Fries et al., 2001). Interestingly, this increase in coherence occurs in the upper layers of the cortex but not in the lower layers (Buffalo et al., 2004) (Fig. 1). Additional support for the attention hypothesis comes from recordings from monkeys trained to perform visual search tasks in which the monkey is cued with a color or shape and asked to find a target with the cued feature amid a large number of distracters (Bichot et al., 2005). When a stimulus with a cued feature appears in the receptive field of a neuron that responds preferentially to that feature, the coherence increases between its spikes and local field potential in the gamma band.

This link between attention and spike-field coherence raises a number of interesting questions. How does the top-down input regulate the coherence of neurons in a cortical column? How is the rapidity of the shifts in coherence achieved? Experimental studies on rodent cortex implicate inhibitory interneuron networks as sources of the oscillations and potential targets of topdown inputs. In particular, excitatory input can rapidly synchronize a subset of the inhibitory neurons that are in competition with other inhibitory networks (Tiesinga and Sejnowski, 2004). Interestingly, a moderate level of background activity is needed to achieve both rapid onsets and offsets. Furthermore, this model can explain why in some experiments there is a shift in sensitivity of the firing rate versus current relationship rather than a gain of the response, depending on whether or not the response function saturates at high currents (Tiesinga et al., 2004, 2005).

An increase in the local coherence in a population of neurons can boost their firing rates as well as the downstream neurons, giving them a competitive advantage (Reynolds and Chelazzi, 2004), as shown in Figure 2. This suggests a "small-world" network architecture (Watts and Strogatz, 1998) in which a few highly active local-circuit interneurons generate local oscillatory synchrony relative to which the more sparsely firing projecting cells communicate information.

\section{Spike timing and oscillations in learning and memory}

A mechanism that regulates spike timing would have some interesting implications for learning and memory. Correlations in input activity can induce synaptic plasticity (Bliss and Lomo, 1973; McNaughton and Douglas, 1978; Golding et al., 2002); if the salient inputs were correlated, they would automatically in-
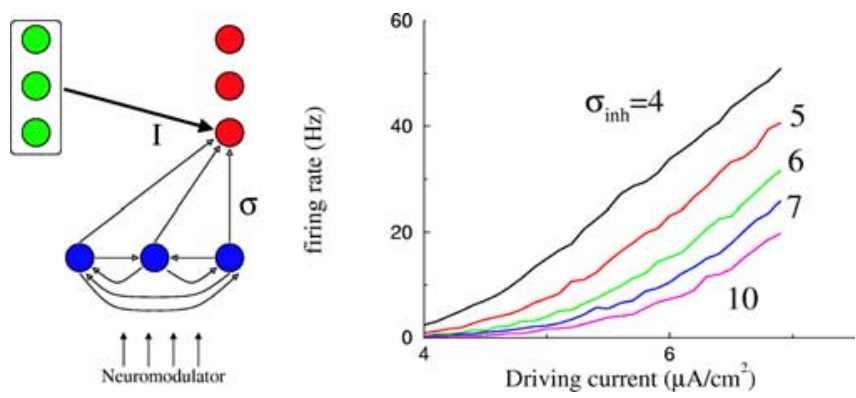

Figure 2. Gain is regulated by synchrony. A Hodgkin-Huxley model cortical neuron (red) receives tuned excitatory input I from neurons (green) in an earlier stage of processing and $40 \mathrm{~Hz}$ oscillatory inhibitory inputs from local interneurons (blue). The timing of the inhibitory input spikes, $\sigma_{\text {inh }}$, is jittered by $4-10 \mathrm{~ms}$. The firing rate versus input current plots are shown in the right panel for different values of the jitter. As the jitter is decreased from 10 to $4 \mathrm{~ms}$, the gain of the responses to the excitatory inputs are increased by factor of 10 . The amount of jitter can be regulated by top-down excitatory inputs or by neuromodulation. Modified from Tiesinga et al. (2004).

crease the likelihood that this information would be stored in memory. Thus, a simple biophysical mechanism could explain why attention enhances learning, without any need to postulate neuromodulator effects on the plasticity process per se.

There is increasing evidence that information is stored in the brain by changes in synaptic weights governed by plasticity rules similar to those originally formulated by Donald Hebb (Hebb, 1949; Sejnowski, 1977; Sejnowski, 1999). Recently, it was discovered that the sign of synaptic plasticity depends on the millisecond precision spike order of presynaptic and postsynaptic neurons (Markram et al., 1997). Such rules have been termed spike-timing-dependent plasticity (STDP) (Song et al., 2000). By naturally organizing the presynaptic and postsynaptic spike times, network oscillations are conducive to STDP (Paulsen and Sejnowski, 2000) and could implement predictive sequence learning in recurrent networks (Montague and Sejnowski, 1994; Rao and Sejnowski, 2003).

If information is stored during oscillations via an STDP rule, how is this information most efficiently retrieved? A theoretical 
argument has been made recently based on Bayesian inference, that information stored as changes of synaptic weights in a recurrent network via an STDP rule would be optimally retrieved via specific spike-timing-based interactions between neurons (Lengyel et al., 2005). The phase response curves of hippocampal pyramidal neurons were indeed found to be matched to the form of hippocampal STDP and thus to be ideally suited for memory retrieval: they can show both advancement and delay of spike times by a preceding excitatory synaptic input (Lengyel et al., 2005). Whether the brain uses such mechanisms to store and retrieve information remains to be experimentally tested.

Finally, let us consider how sensory information is routed to the correct motor outputs after an instruction to press a button when a target dims. There must be an internal system in the brain that controls attention, expectation, and memory that can be flexibly and rapidly reconfigured. In addition to the relatively fixed network of connections, the brain needs a fast way to reorganize the communications channels that link different cortical areas. The evidence summarized here suggests that the oscillations observed in the EEG and local field potentials are a reflection of this communications system and that spike timing is used to regulate the flow and storage of information in cortical circuits. If this is the case, then EEG recordings may provide insights into how the reconfiguration is accomplished.

In addition to the evidence for the involvement of gammaband oscillations in the local control of attention and memory, which was the focus of this mini-symposium, oscillations in the theta band as reviewed by Kahana (2006) may reflect an even larger-scale system of communications and control that becomes engaged during more complex exploratory behaviors and cognitive tasks. New techniques for analyzing single-trial event-related potentials and functional magnetic resonance signals may help us to eavesdrop on the communications system of the brain (Jung et al., 2001; Laughlin and Sejnowski, 2003; McKeown et al., 2003).

\section{Synthesis}

Network oscillations are a prominent feature of neuronal activity throughout the animal kingdom. In the primate brain, there is growing evidence that oscillations are linked to behavior and cognitive tasks. Because action potential generation is energetically expensive, this mechanism may have evolved to minimize energy expenditure during computation and communication (Laughlin and Sejnowski, 2003), which might also explain why it is evolutionarily conserved from mollusks to the human brain (Gelperin, 2006; Kahana, 2006).

\section{References}

Bazhenov M, Stopfer M, Rabinovich M, Huerta R, Abarbanel HD, Sejnowski TJ, Laurent G (2001a) Model of transient oscillatory synchronization in the locust antennal lobe. Neuron 30:553-567.

Bazhenov M, Stopfer M, Rabinovich M, Abarbanel HD, Sejnowski TJ, Laurent G (2001b) Model of cellular and network mechanisms for odorevoked temporal patterning in the locust antennal lobe. Neuron 30:569-581.

Bichot NP, Rossi AF, Desimone R (2005) Parallel and serial neural mechanisms for visual search in macaque area V4. Science 308:529-534.

Bliss TV, Lomo T (1973) Long-lasting potentiation of synaptic transmission in the dentate area of the anaesthetized rabbit following stimulation of the perforant path. J Physiol (Lond) 232:331-356.

Brody CD, Hopfield JJ (2003) Simple networks for spike-timing-based computation, with application to olfactory processing. Neuron 37:843-852.

Buffalo EA, Fries P, Desimone R (2004) Layer-specific attentional modulation in early visual areas. Soc Neurosci Abstr 30:717.6.

Buracas GT, Zador AM, DeWeese MR, Albright TD (1998) Efficient dis- crimination of temporal patterns by motion-sensitive neurons in primate visual cortex. Neuron 20:959-969.

Fellous J-M, Houweling AR, Modi RH, Rao RP, Tiesinga PH, Sejnowski TJ (2001) Frequency dependence of spike timing reliability in cortical pyramidal cells and interneurons. J Neurophysiol 85:1782-1787.

Fisahn A, Pike FG, Buhl EH, Paulsen O (1998) Cholinergic induction of network oscillations at $40 \mathrm{~Hz}$ in the hippocampus in vitro. Nature 394:186-189.

Fries P, Reynolds JH, Rorie AE, Desimone R (2001) Modulation of oscillatory neuronal synchronization by selective visual attention. Science 291:1560-1563.

Gelperin A (2006) Olfactory computations and network oscillations. J Neurosci 26:1663-1668.

Golding NL, Staff NP, Spruston N (2002) Dendritic spikes as a mechanism for cooperative long-term potentiation. Nature 418:326-331.

Gray CM, Singer W (1989) Stimulus-specific neuronal oscillations in orientation columns of cat visual cortex. Proc Natl Acad Sci USA 86:1698-1702.

Harris KD, Henze DA, Hirase H, Leinekugel X, Dragoi G, Czurko A, Buzsaki G (2002) Spike train dynamics predicts theta-related phase precession in hippocampal pyramidal cells. Nature 417:738-741.

Hebb DO (1949) The organization of behavior. New York: Wiley.

Hopfield JJ (1995) Pattern recognition computation using action potential timing for stimulus representation. Nature 376:33-36.

Huxter J, Burgess N, O’Keefe J (2003) Independent rate and temporal coding in hippocampal pyramidal cells. Nature 425:828-832.

Jensen O, Lisman JE (2000) Position reconstruction from an ensemble of hippocampal place cells: contribution of theta phase coding. J Neurophysiol 83:2602-2609.

Jung T-P, Makeig S, McKeown MJ, Bell AJ, Lee T-W, Sejnowski TJ (2001) Imaging brain dynamics using independent component analysis. Proc IEEE 89:1107-1122.

Kahana MJ (2006) The cognitive correlates of human brain oscillations. J Neurosci 26:1669-1672.

Kara P, Reinagel P, Reid RC (2000) Low response variability in simultaneously recorded retinal, thalamic, and cortical neurons. Neuron 27:635-646.

Laughlin SB, Sejnowski TJ (2003) Communication in neuronal networks. Science 301:1870-1874.

Lengyel M, Kwag J, Paulsen O, Dayan P (2005) Matching storage and recall: hippocampal spike timing-dependent plasticity and phase response curves. Nat Neurosci 8:1677-1683.

Mainen ZF, Sejnowski TJ (1995) Reliability of spike timing in neocortical neurons. Science 268:1503-1506.

Markram H, Lubke J, Frotscher M, Sakmann B (1997) Regulation of synaptic efficacy by coincidence of postsynaptic APs and EPSPs. Science 275:213-215.

McKeown MJ, Hansen LK, Sejnowski TJ (2003) Independent component analysis of functional MRI: what is signal and what is noise? Curr Opin Neurobiol 13:620-629.

McNaughton BL, Douglas RM (1978) Synaptic enhancement in fascia dentata: cooperativity among coactive afferents. Brain Res 157:277-293.

Mehta MR, Lee AK, Wilson MA (2002) Role of experience and oscillations in transforming a rate code into a temporal code. Nature 417:741-746.

Mel BW (1999) Why have dendrites? A computational perspective. In: Dendrites (Stuart G, Spruston N, Hausser M, eds), pp 271-289. Oxford: Oxford UP.

Montague PR, Sejnowski TJ (1994) The predictive brain: temporal coincidence and temporal order in synaptic learning mechanisms. Learn Mem $1: 1-33$.

Nowak LG, Sanchez-Vives MV, McCormick DA (1997) Influence of low and high frequency inputs on spike timing in visual cortical neurons. Cereb Cortex 7:487-501.

O'Keefe J, Recce ML (1993) Phase relationship between hippocampal place units and the EEG theta rhythm. Hippocampus 3:317-330.

Paulsen O, Sejnowski TJ (2000) Natural patterns of activity and long-term synaptic plasticity. Curr Opin Neurobiol 10:172-179.

Pike FG, Goddard RS, Suckling JM, Ganter P, Kasthuri N, Paulsen O (2000) Distinct frequency preferences of different types of rat hippocampal neurones in response to oscillatory input currents. J Physiol (Lond) 529:205-213. 
Rao RP, Sejnowski TJ (2003) Self-organizing neural systems based on predictive learning. Philos Transact A Math Phys Eng Sci 361:1149-1175.

Reynolds JH, Chelazzi L (2004) Attentional modulation of visual processing. Annu Rev Neurosci 27:611-647.

Rieke F, Warland D, de Ruyter van Steveninck RR, Bialek W (1997) Spikes: exploring the neural code. Cambridge, MA: MIT.

Salinas E, Sejnowski TJ (2000) Impact of correlated synaptic input on output firing rate and variability in simple neuronal models. J Neurosci 20:6193-6209.

Salinas E, Sejnowski TJ (2001) Correlated neuronal activity and the flow of neural information. Nat Rev Neurosci 2:539-550.

Schoffelen JM, Oostenveld R, Fries P (2005) Neuronal coherence as a mechanism of effective corticospinal interaction. Science 308:111-113.

Sejnowski TJ (1977) Storing covariance with nonlinearly interacting neurons. J Math Biol 4:303-321.

Sejnowski TJ (1999) The book of Hebb. Neuron 24:773-776.

Shadlen MN, Newsome WT (1998) The variable discharge of cortical neurons: implications for connectivity, computation, and information coding. J Neurosci 18:3870-3896.

Singer W, Gray CM (1995) Visual feature integration and the temporal correlation hypothesis. Annu Rev Neurosci 18:555-586.
Softky WR (1995) Simple codes versus efficient codes. Curr Opin Neurobiol 5:239-247.

Song S, Miller KD, Abbott LF (2000) Competitive Hebbian learning through spike-timing-dependent synaptic plasticity. Nat Neurosci 3:919-926.

Tiesinga PHE, Sejnowski TJ (2004) Rapid temporal modulation of synchrony by competition in cortical interneuron networks. Neural Comput 16:251-275.

Tiesinga PHE, Fellous J-M, Salinas E, Jose JV, Sejnowski TS (2004) Synchronization as a mechanism for attentional gain modulation. Neurocomputing 58-60:641-646.

Tiesinga PHE, Fellous J-M, Salinas E, Jose JV, Sejnowski TJ (2005) Inhibitory synchrony as a mechanism for attentional gain modulation. J Physiol (Paris) 98:296-314.

Tsodyks MV, Skaggs WE, Sejnowski TJ, McNaughton BL (1996) Population dynamics and theta rhythm phase precession of hippocampal place cell firing: a spiking neuron model. Hippocampus 6:271-280.

Watts DJ, Strogatz SH (1998) Collective dynamics of "small-world" networks. Nature 393:440-442.

Zhang K, Ginzburg I, McNaughton BL, Sejnowski TJ (1998) Interpreting neuronal population activity by reconstruction: unified framework with application to hippocampal place cells. J Neurophysiol 79:1017-1044. 